\title{
Urs Müller-Plantenberg Marktwirtschaft und Demokratie in Lateinamerika
}

Zusammenfossung: Politische Demokratie und marktförmige Organisation der Wirtschaft sind heute die Zauberformeln, die den Ländern Afrikas, Asiens und Lateinamerika zur Lösung ihrer Entwicklungsprobleme angeboten werden. Gezeigt wird, daß die behauptete Harmonie von Demokratie und Marktwirtschaft weder selbstverständlich ist noch in großen Teilen der Welt die erforderlichen Rahmenbedingungen gegeben sind, um beide Prinzipien erfolgreich miteinander kombinieren zu können.

Der politische Umbruch, der im Jahr 1989 in Osteuropa einen breiten Prozeß der politischen Demokratisierung und wirtschaftlichen Liberalisierung freigesetzt hat, laßt die Demokratisierungsprozesse der achtziger Jahre in Lateinamerika im nachhinein als Vorläufer und Ausdruck einer weltweiten Tendenz erscheinen. Marktwirtschaft und Demokratie, wie sie sich in den letzten Jahrzehnten in den entwickelten kapitalistischen Industrienationen Westeuropas, Nordamerikas und Ostasiens durchgesetzt haben, sind zu den Zwillingssternen geworden, die den Ländern Asiens, Afrikas und Lateinamerikas in der Finsternis leuchten sollen. Schon ist die Rede vom erlösenden Ende der Geschichte, das kommen könnte, wenn alle Länder und Regionen der Zweiten und Dritten Welt das einzig akzeptable Modell aus der Ersten Welt als verpflichtend anerkannt hätten.

Dabei wird darauf vertraut, daß demokratisch legitimierte Herrschaft und das Wirken von Marktmechanismen in der Wirtschaft überall auf der Welt soziale Gerechtigkeit befördern und ökologische Vernunft bewirken könnten, wenn sie nur sozial und ökologisch orientiert seien. Sozial und ökölogisch orientierte Demokratie und Marktwirtschaft: das ist überhaupt der Schlüssel zum Paradies.

Das Problem besteht darin, daß die behauptete Harmonie dieser Zielfunktionen keineswegs selbstverständlich ist und daß das gemeinsame Funktionieren von Demokratie und Marktwirtschaft mit wirksamer sozialer und ökologischer Orientierung von Rahmenbedingungen abhängt, die in großen Teilen der Welt nicht gegeben sind. Das soll im folgenden Beitrag in Ansätzen am Beispiel Lateinamerikas gezeigt werden, wo die soziale und ökologische Orientierung so real existiert wie einst der Sozialismus in Osteuropa.

\section{Demokratie und Markt: Äquivalenz oder Spannung?}

Zunächst muß aber betont werden, daß Demokratie und Marktwirtschaft keineswegs das Zwillingsgespann sind, als das sie gewöhnlich dargestellt werden. Orthodoxe Liberale als die geborenen Ideologen des Marktes und demokratische So- 
zialisten als Vorkämpfer der Demokratie wissen sehr gut, daß sich beide aus ganz verschiedenen Wurzeln, Traditionen und Interessen speisen. Tatsächlich erweisen sich die Hinweise auf die Åquivalenz von Demokratie und Markt als komplexen, pluralistischen Formen des Wettbewerbs in einer offenen Ordnung sowie auf ihre Komplementarităt und Interdependenz durch die gegenseitigen Freiheitsgarantien als teilweise ausschließlich formal, teilweise offen ideologisch. Friedrich August von Hayek hat theoretisch nachgewiesen, was der langjährige chilenische Diktator Augusto Pinochet dann praktisch gezeigt hat, daß sich nämlich die Durchsetzung und Geltung einer konsequent marktwirtschaftlichen Ordnung durchaus mit einer extrem autoritären und antidemokratischen politischen Ordnung verträgt, diese sogar zur Voraussetzung haben kann, wie andererseits - auch nach von Hayek - demokratisch legitimierte Herrschaft möglich ist, ohne daß der Markt seine Wirkungen voll entfaltet.

Aus vielerlei Gründen war den verbissenen Liberalen die Demokratie schon immer unheimlich. Demokratie bedeutet zunächst einmal Politik: Demokraten maßen sich - so schon der Vorwurf des orthodoxen Liberalismus gegen den Sündenfall des Gesetzes über den Zwölfstundentag - $\mathrm{an}_{\text {, }}$ in das freie Leben der Gesellschaft und vor allem der Wirtschaft politisch einzugreifen. Demokratie bedeutet weiterhin das Bemühen um kollektive Entscheidungen: Demokraten maßen sich an, sich über die freien Entscheidungen der Individuen und vor allem der Wirtschaftssubjekte gemeinsam hinwegzusetzen. Und schließlich bedeutet Demokratie eine Begünstigung der Mehrheit: Demokraten dulden oder begrüßen es gar, daß den Interessen der zahlenmäßigen Mehrheit mehr Rechnung getragen wird als der zahlungsfähigen Nachfrage. An die Stelle des Geldes als allgemeines Äquivalent eine Mark ist eine Mark - soll plötzlich das Prinzip treten: ein Mensch, eine Stimme, egal über wieviel Mark dieser Mensch verfügt.

\section{Der Sozialismusverdacht gegen die Demokratie}

Die Pareto-Regel als oberstes Prinzip der liberalen Wohlfahrtsokonomie, wonach nämlich eine Handlung nur dann optimal ist, wenn sie niemandem schadet, aber mindestens einem Beteiligten nützt, und das Mehrheitsprinzip als demokratisches Prinzip sind unvereinbar wie Feuer und Wasser. Wo Demokratie überhaupt wirksam wird, greift sie zugunsten der Mehrheit und im Dienste sozialer Gerechtigkeit in den freien Markt ein, setzt sie ihm Grenzen, reguliert sie ihn und tangiert daher zwangsläufig auch bestimmte Interessen. Insofern erscheint eine funktionierende Demokratie den Liberalen bereits als das Schlimmste, was sie sich vorstellen können: als Sozialismus. Die Militärputsche, die zwischen 1964 und 1976 in Südamerika die mehr oder weniger demokratischen Regierungen Brasiliens, Boliviens, Uruguays, Chiles und Argentiniens hinwegfegten, wurden deshalb von ihnen wenn schon nicht gefeiert, so doch als leider notwendige antisozialistische $» \mathrm{Be}$ freiungsaktionen « begrüßt.

Wo sich die Ideologen des Marktes auf Demokratie als das noch geringste aller 
politischen Übel einlassen, werden sie stets versuchen, ihren Inhalt auf Repråsentation und formale Verfahren zu reduzieren und ihren Anspruch auf Herrschaft der Mehrheit und Durchsetzung sozialer Gerechtigkeit abzuwehren. Was an sozialer Gerechtigkeit nötig und möglich ist, stellt sich nach ihrer Auffassung zwangslåufig als Nebenergebnis der Bewegungen des Marktes ein. Andersherum wollen Demokraten am liebsten gar nicht zur Kenntnis nehmen, daß der kapitalistische Markt Mehrwertaneignung und Kapitalakkumulation zur Voraussetzung hat, und betonen stattdessen nur die förderlichen Wirkungen des freien Wettbewerbs.

Nun steht Demokratie nicht umsonst unter dem Sozialismusverdacht. Was immer in den kapitalistischen Industriegesellschaften an sozialem Fortschritt und sozialer Gerechtigkeit gegen den Widerstand der Manchesterkapitalisten und konservativer Feudalherren erreicht worden ist, konnte nur in dem Maße erkämpft und gesichert werden, wie gleichzeitig die Demokratie als politisches System erkämpft und gesichert wurde. Umverteilung zugunsten der zahlenmäßig starken, aber ökonomisch schwachen Schichten kann dauerhaft nur wirksam sein, wo anerkannt ist, da $B$ die Mehrheit das Recht hat, in einem kollektiven Entscheidungsproze $B$ ihre Interessen durchzusetzen. Es ist daher auch nicht erstaunlich, daß die internationale Arbeiterbewegung Demokratie immer als eine Voraussetzung für Sozialismus und diesen als die Vollendung der Demokratie begriffen hat.

Der real existierende Sozialismus osteuropäischer Prägung hat diesen Anspruch, Vollendung der Demokratie zu sein, durchaus aufrechterhalten. Aber er hat die Beweisführung einfach umgedreht, um sich diesen Vorzug möglichst lange in die Tasche lügen zu können: Schon weil eine politische Entscheidung im vorgestellten Interesse oder auch nur im Namen einer strukturellen Mehrheit gefällt wurde, konnte sie nach dem dort geltenden Schema als sozialistisch und damit auch als demokratisch gelten. Schon weil das System den Kräften des Marktes keinen Raum lie $B$ und alle Handlungen als bewußte politische Maßnahmen wertete und einem Plan unterordnete, glaubte es, den Anspruch auf Verwirklichung des Sozialismus und daraus dann auch noch den Anspruch auf Vollendung der Demokratie ableiten zu können. Es ist gerade diese Anmaßung, die bei der ersten empirischen Befragung des wirklich existierenden Volkswillens in den meisten Ländern Osteuropas zum Sturz des Systems geführt und die verheerende Diskreditierung des Begriffs Sozialismus offengelegt hat.

Spätestens seither hat sich das Generalthema der weltweiten politisch-ökonomischen Debatte gründlich verschoben. Statt eines Kampfes zwischen den extremen Polen eines Manchesterkapitalismus einerseits und einer alle Marktmechanismen ablehnenden Planwirtschaft gibt es, sieht man von Kuba $a b$, nur noch die allgemeine Akzeptanz der Marktwirtschaft. Und noch mehr: Auch daß sie sozial und ökologisch orientiert sein muß, ist von Alaska bis Kamtschatka, von Spitzbergen bis Feuerland völlig unumstritten. Der Streit geht nur noch darum, was das denn nun im einzelnen heißen soll: sozial und ökologisch orientiert.

Diese Debatte ist auf merkwuirdige Weise einförmig geworden. Als ob die Welt bereits eine einzige geworden sei, dreht sich die Diskussion in allen Ländern, ob 
reich, ob arm, ob stark, ob schwach, nur um die scheinbar überall gleiche Frage nach dem grundsätzlich richtigen Ausmaß der Regulierung oder Deregulierung. Dabei wird übersehen, daß in einem armen, unterentwickelten, verschuldeten Land im Rahmen der Marktwirtschaft mit keinem Grad von Regulierung oder Deregulierung auch nur ein Bruchteil dessen erreicht werden kann, was etwa in der Bundesrepublik Deutschland an sozialer Gerechtigkeit und okologischer Vernunft im Prinzip durchsetzbar und finanzierbar wäre.

Es gibt eben nicht eine einzige, weltweite Marktwirtschaft, über deren soziale und okologische Orientierung weltweit gestritten werden könnte, sondern es gibt viele verschiedene Marktwirtschaften, die mit den internationalen Märkten für Waren, Dienstleistungen, Kapital, Technologien und Arbeitskräfte in unterschiedlichem Ausmaß verbunden sind.

\section{Verteilungsspielraum in Lateinamerika}

Ausschlaggebend für die Frage, welche Möglichkeiten die einzelnen Lånder überhaupt haben, durch Sozialleistungen und Umweltschutzmaßnahmen soziale Gerechtigkeit und okologische Vernunft zu befördern, ist zunächst einmal der Verteilungsspielraum, der sich aus der Höhe der Produktion ergibt. Tabelle 1 zeigt, daß die meisten Länder Lateinamerikas und der Karibik in den achtziger Jahren eindeutig zu den Verlierem der Weltwirtschaft gehört haben. In einem Jahrzehnt, in dem das Wachstum des Bruttoinlandsprodukts pro Kopf der Bevolkerung in Japan um über 40 Prozent, in den USA um über 30 und in den Låndern der Europåischen Gemeinschaft um über 20 Prozent gestiegen ist, gab es unter den aufgeführten 20 Ländem gerade zwei, die überhaupt ein bescheidenes Wachstum aufweisen konnten, nämlich Kolumbien mit 16 und Chile mit 9 Prozent innerhalb des ganzen Jahrzehnts. Und dabei ist zu berücksichtigen, daß dieses Wachstum in Kolumbien im wesentlichen dem durch den illegalen Drogenhandel induzierten Boom zu danken ist, während Chile nur aufgeholt hat, was es schon vor 1980 verloren hatte.

Dann gibt es mit Jamaika und Paraguay noch zwei Länder, in denen 1990 das Niveau von 1980 gerade wieder erreicht werden konnte, und alle anderen Länder haben schwere Einbußen hinnehmen müssen. In Nicaragua sank die Produktion pro Kopf der Bevölkerung im letzten Jahrzehnt um mehr als 40 Prozent, in Peru um mehr als 30, in Argentinien um 24, in Bolivien um 23, in Haiti um 22, in Venezuela um 20, in Panama und Guatemala um 18 Prozent. In der gesamten Region sank das Bruttoinlandsprodukt pro Kopf der Bevölkerung in den achtziger Jahren um knapp 10 Prozent. Die Jahre geringen Wachstums von 1984 bis 1987 reichten längst nicht aus, um die Verluste der Krise von 1981 bis 1983 auszugleichen, und die Jahre 1988 bis 1990 bedeuteten dann einen weiteren Rückschritt in einer Periode, in der die wichtigsten Industrienationen der sogenannten Ersten Welt ihr Wirtschaftswachstum sogar noch steigern konnten. 
Tabelle 1 Lateinamerika und Karibik:

Bruttoinlandsprodukt pro Eimwohner 1980 bis 1990 (in US-Dollar von 1987)

$\begin{array}{lrrrrrrrr}\text { Land } & 1980 & 1982 & 1984 & 1986 & 1987 & 1988 & 1989 & 1990 \\ \text { Venezuela } & 3841 & 3549 & 3122 & 3157 & 3230 & 3338 & 3019 & 3076 \\ \text { Argentinien } & 2763 & 2351 & 2404 & 2371 & 2390 & 2290 & 2161 & 2092 \\ \text { Uruguay } & 2350 & 2117 & 1939 & 2070 & 2190 & 2181 & 2197 & 2192 \\ \text { Panama } & 2168 & 2264 & 2158 & 2242 & 2240 & 1843 & 1793 & 1772 \\ \text { Mexiko } & 2004 & 2065 & 1954 & 1842 & 1830 & 1814 & 1828 & 1836 \\ \text { Brasilien } & 1998 & 1836 & 1782 & 1990 & 2020 & 1976 & 2006 & 1888 \\ \text { Costa Rica } & 1769 & 1511 & 1578 & 1582 & 1610 & 1619 & 1665 & 1681 \\ \text { Peru } & 1522 & 1510 & 1321 & 1395 & 1470 & 1318 & 1146 & 1062 \\ \text { Chile } & 1367 & 1224 & 1220 & 1269 & 1310 & 1387 & 1491 & 1493 \\ \text { Guatem. } & 1182 & 1089 & 1002 & 943 & 950 & 959 & 968 & 969 \\ \text { Ecuador } & 1180 & 1170 & 1147 & 1179 & 1040 & 1170 & 1138 & 1126 \\ \text { Kolumbien } & 1119 & 1108 & 1124 & 1197 & 1240 & 1261 & 1279 & 1300 \\ \text { Paraguay } & 1052 & 1065 & 1001 & 976 & 990 & 1026 & 1055 & 1056 \\ \text { Nicaragua } & 1034 & 1012 & 975 & 864 & 830 & 714 & 671 & 612 \\ \text { El Salvador } & 1005 & 850 & 859 & 853 & 860 & 855 & 846 & 851 \\ \text { Jamaika } & 976 & 969 & 941 & 891 & 940 & 939 & 987 & 995 \\ \text { Honduras } & 916 & 842 & 802 & 801 & 810 & 824 & 817 & 786 \\ \text { Bolivien } & 763 & 699 & 631 & 579 & 580 & 583 & 584 & 585 \\ \text { Dominik. Rep. } & 712 & 714 & 717 & 694 & 730 & 722 & 739 & 697 \\ \text { Haiti } & 428 & 388 & 378 & 368 & 360 & 352 & 346 & 333\end{array}$

Lateinam. u. Karibik

$\begin{array}{lllllllll}\text { insgesamt } & 1894 & 1792 & 1727 & 1775 & 1790 & 1769 & 1758 & 1712 \\ \text { BRD } & & & & & 14400 & & & \\ \text { Schweiz } & & & & & 21330 & & & \end{array}$

Die Angaben für 1990 sind vorläufige Schätzungen.

Quellen: für das absolute Niveau 1987: Weltentwicklungsbericht 1989, Weltbank, Wahington 1989, für die jährlichen Veränderungen: CEPAL ( Wirtschaftskommission der Vereinten Nationen für Lateinamerika und die Karibik ), Notas sobre la economía y el desarrollo, Nr. 500/501, Santiago 1990.

Zu behaupten, daß diese enormen Rückgånge wirtschaftlicher Aktivität sozialisierenden Tendenzen oder auch nur einem Mangel an Markttransparenz geschuldet seien, ist schlicht und einfach falsch. In jedem einzelnen der Fälle trat der entscheidende Rückgang der Produktion erst als Folge eines beschleunigten Übergangs zu einer neoliberalen, marktorientierten Wirtschaftspolitik ein, meist im Zu- 
sammenhang mit den vom Internationalen Wăhrungsfonds (IWF) geforderten und von der Weltbank geförderten Strukturanpassungsprogrammen. Das kann man ohne weiteres aus den Daten der Tabelle 1 für Venezuela und Argentinien 1989, Panama 1988, Peru und Nicaragua 1988 bis 1990 oder Bolivien 1985 ablesen.

\section{Die Auslandsverschuldung}

Insgesamt ist festzuhalten, daß die durch die Produktion gesetzten Grenzen für den Verteilungsspielraum, den eine sozial und ökologisch orientierte Demokratie benotigt, im letzten Jahrzehnt in Lateinamerika immer enger geworden sind. Diese Grenzen werden aber noch einmal wesentlich enger gezogen durch die Situation der Auslandsverschuldung, in der sich alle lateinamerikanischen Länder befinden und die dem IWF und der Weltbank erst erlauben, ihre Programme für die Umstrukturierung der Wirtschaft in diesen Ländern durchzusetzen.

Die Tabelle 2 zeigt die Entwicklung der Auslandsverschuldung der wichtigsten lateinamerikanischen Lånder in den letzten sechs Jahren. Bis auf Mexiko, wo der groß angekündigte Schuldensenkungsplan von US-Finanzminister Brady 1989/90 gerade mal einen Rückgang der Auslandsschulden um fünf Prozent erbracht hat, Chile, wo die Militärdiktatur kurz vor ihrem Ende 1988/89 die Schulden noch durch eine Politik des Ausverkaufs von nationalem Produktionspotential um 15 Prozent senken konnte, und schließlich Venezuela, Bolivien und Costa Rica haben alle anderen lateinamerikanischen Länder in den Jahren von 1985 bis 1990 eine leichte oder sogar starke Steigerung der Auslandsschulden erlebt.

Nun könnte man denken, daß die Aufnahme neuer Schulden eine Ausweitung des Verteilungsspielraums und damit eine wenigstens relative oder zeitweilige Erleichterung für eine sozial und §kologisch orientierte Politik bieten müßte. Aber das Gegenteil ist richtig.

Seit 1982 haben die relativ weniger armen Länder Lateinamerikas nicht nur keinen Nettozufluß an Ressourcen erlebt, sondern im Gegenteil durch Zahlungen von Zinsen und Gewinnen in Höhe von insgesamt 318,5 Milliarden US-Dollar, die nur zu geringen Teilen durch Neuaufnahme von 94,9 Milliarden Kapital finanziert werden konnten, einen gigantischen Ressourcentransfer von 223,6 Milliarden USDollar in die westlichen Industrieländer finanzieren müssen, der real ungefähr dem Dreifachen dessen entspricht, was mit dem Marshall-Plan nach dem Zweiten Weltkrieg an Hilfsgütern aus den USA nach Europa geflossen ist. Mit diesen Zahlungen haben aber die Auslandsschulden der Region in der gleichen Zeit keineswegs abgenommen, sondern sogar noch um insgesamt 125 Milliarden USDollar zugenommen (vgl. Hinkelammert 1989).

Bei diesem Ressourcentransfer handelt es sich nicht etwa um fiktive Summen, die in den Büchern hin- und hergeschoben werden, sondern um reale Güter und Dienstleistungen, die ohne Gegenleistungen aus Lateinamerika abgezogen werden. Die Gegenleistung besteht allenfalls darin, daß es den lateinamerikani 
Tabelle 2 Lateinamerika und Karibik:

Gesamtauslandsschuld 1985 bis 1990

(Jahresendsalden in Milliarden US-Dollar)

$\begin{array}{lrrrrrr}\text { Land } & 1985 & 1986 & 1987 & 1988 & 1989 & 1990 \\ \text { Brasilien } & 105,1 & 111,0 & 121,2 & 113,5 & 115,1 & 121,0 \\ \text { Mexiko } & 97,8 & 100,5 & 102,4 & 100,4 & 95,1 & 95,9 \\ \text { Argentinien } & 49,3 & 51,4 & 58,3 & 58,5 & 63,3 & 67,5 \\ \text { Venezuela } & 33,4 & 32,9 & 34,2 & 34,3 & 32,0 & 31,0 \\ \text { Peru } & 13,7 & 14,5 & 15,4 & 16,5 & 16,7 & 17,7 \\ \text { Kolumbien } & 14,1 & 15,0 & 15,7 & 16,4 & 16,2 & 17,2 \\ \text { Chile } & 20,4 & 20,7 & 20,7 & 17,6 & 16,3 & 16,9 \\ \text { Ecuador } & 8,1 & 9,1 & 10,2 & 10,6 & 11,0 & 11,7 \\ \text { Nicaragua } & 5,0 & 5,8 & 6,3 & 7,2 & 8,1 & 8,6 \\ \text { Uruguay } & 4,9 & 5,2 & 5,9 & 6,3 & 7,0 & 6,9 \\ \text { Panama } & 4,8 & 4,9 & 5,3 & 5,4 & 5,5 & 5,8 \\ \text { Dominik. Rep. } & 3,7 & 3,8 & 3,9 & 3,9 & 4,1 & 4,3 \\ \text { Jamaika } & 3,4 & 3,6 & 4,0 & 4,0 & 4,0 & 4,0 \\ \text { Bolivien } & 3,3 & 3,5 & 4,2 & 4,1 & 3,5 & 3,7 \\ \text { Honduras } & 2,8 & 3,0 & 3,1 & 3,3 & 3,4 & 3,6 \\ \text { Costa Rica } & 3,7 & 3,9 & 4,2 & 4,1 & 3,8 & 3,0 \\ \text { Guatemala } & 2,7 & 2,7 & 2,7 & 2,6 & 2,7 & 2,8 \\ \text { El Salvador } & 2,0 & 1,9 & 1,9 & 1,9 & 2,2 & 2,2 \\ \text { Paraguay } & 1,8 & 1,9 & 2,0 & 2,0 & 2,0 & 2,1 \\ \text { Haiti } & 0,6 & 0,7 & 0,8 & 0,8 & 0,8 & 0,8 \\ \text { Lateinamerika u. Karibik } & & & & & \\ \text { insgesamt } & 383,5 & 399,4 & 426,0 & 417,9 & 417,5 & 422,6 \\ & & & & & & \end{array}$

Die Angaben für 1990 sind vorläufige Schätzangen.

Quelle: CEPAL, a.a.O., Santiago 1990

schen Ländern erlaubt wird, die Zinsen dafür zu zahlen, daß sie in den siebziger Jahren für ein scheinbar problemloses Recycling einiger Ölmilliarden gesorgt haben.

In der Tabelle 3 kann man ablesen, daß dieser Ressourcentransfer seit 1982 mehr als ein Fünftel der gesamten Ausfuhr von Gütern und Dienstleistungen ausgemacht hat, in manchen Jahren - wie 1983 oder 1985 - sogar fast ein Drittel.

Noch deutlicher wird der Aderlaß, wenn man ihn für einzelne Länder betrachtet und in Beziehung zum gesamten erarbeiteten Bruttoinlandsprodukt dieser Länder setzt. Die in Tabelle 4 aufgeführten Summen ergeben zunächst einmal, daß aus Brasilien und Mexiko in den letzten neun Jahren jeweils Werte herausgeholt wur- 
den, die einen vollen Marshall-Plan ergeben hätten. Das entsprach in Brasilien in den einzelnen Jahren einem Anteil zwischen zwei und fünf Prozent des Bruttoinlandsprodukts. In Kolumbien, Chile und Uruguay ist dieser Anteil in einzelnen Jahren auf sechs, in Mexiko, Venezuela und Argentinien sogar auf acht bis zehn Prozent gestiegen.

Der Verteilungsspielraum, den die lateinamerikanischen Demokratien benörigen würden, um soziale Gerechtigkeit und okologische Vernunft walten zu lassen, wird also nicht nur durch eine - pro Kopf der Bevölkerung - zurückgehende Produktion eingeschrånkt, sondern zusätzlich auch durch die Auslandsverschuldung, die dafür sorgt, daß von dem schrumpfenden Produkt noch einmal ein erheblicher Teil für das Ausland abgezwackt wird. Aus dem verschuldeten Kapitalismus, für den die strukturelle Abhängigkeit von externer Finanzierung ein Begleitphänomen seiner Reproduktion war, ist im Fall der größeren lateinamerikanischen Länder langst ein "Schuldendienst-Kapitalismus geworden, das heiBt: ein Wirtschaftssystem, in dem sämtliche ökonomischen Variablen dem obersten Ziel der Bedienung der Auslandsschulden angepaßt werden (vgl. Ehrke 1986, S. 26).

Tabelle 3 Lateinamerika und Karibik:

Nettokapitalzufuhr und Ressourcentransfer 1980 bis 1990 (in Milliarden US-Dollar bzw. Prozent )

\begin{tabular}{|c|c|c|c|c|c|}
\hline Jahr & $\begin{array}{l}\text { Netto- } \\
\text { kapital- } \\
\text { zufuhr }\end{array}$ & $\begin{array}{l}\text { Netto- } \\
\text { zahlung } \\
\text { von Zinsen } \\
\text { und } \\
\text { Gewinnen }\end{array}$ & $\begin{array}{l}\text { Ressourcen- } \\
\text { transfer }\end{array}$ & $\begin{array}{l}\text { Ausfuhr } \\
\text { von Gutern } \\
\text { und Dienst- } \\
\text { leistungen }\end{array}$ & $\begin{array}{l}\text { Verhälmis } \\
\text { Ressourcen- } \\
\text { transfer (3) zu } \\
\text { Ausfuhr (4) in } \\
\text { Prozent }\end{array}$ \\
\hline & (1) & (2) & (3) & (4) & (5) \\
\hline 1982 & 20,1 & 38,8 & $-18,7$ & 103,0 & 18,2 \\
\hline 1983 & 2,9 & 34,5 & $-31,6$ & 102,4 & 30,9 \\
\hline 1984 & 10,4 & 37,3 & $-26,9$ & 113,8 & 23,6 \\
\hline 1985 & 3,0 & 35,3 & $-32,3$ & 109,0 & 29,6 \\
\hline 1986 & 9,9 & 32,7 & $-22,8$ & 94,7 & 24,1 \\
\hline 1987 & 15,1 & 31,4 & $-16,3$ & 108,1 & 15,1 \\
\hline 1988 & 5,5 & 34,3 & $-28,8$ & 122,8 & 23,5 \\
\hline 1989 & 10,1 & 37,4 & $-27,3$ & 136,4 & 20,0 \\
\hline 1990 & 17,9 & 36,8 & $-18,9$ & 147,1 & 12,8 \\
\hline Summe $82-90$ & 94,9 & 318,5 & $-223,6$ & 1037,3 & 21,6 \\
\hline
\end{tabular}

Die Angaben für 1990 sind vorläufige Schåızungen.

Quelle: CEPAL, a.a.O., Santiago 1990 
Tabelle 4 Lateinamerika und Karibik:

Ressourcentransfer 1982 bis 1990 (in Milliarden US-Dollar)

$\begin{array}{lrrrrrrrr}\text { Land } & 1984 & 1985 & 1986 & 1987 & 1988 & 1989 & 1990 & 82-90 \\ \text { Brasilien } & -6,1 & -11,4 & -9,9 & -6,7 & -14,5 & -11,7 & -9,3 & -78,0 \\ \text { Mexiko } & -12,1 & -12,0 & -5,3 & -5,2 & -11,3 & -2,4 & 0,7 & -69,6 \\ \text { Venezuela } & -5,2 & -3,6 & -3,6 & -1,3 & 0,2 & -4,8 & -6,4 & -36,4 \\ \text { Argentinien } & -3,1 & -3,3 & -2,2 & -2,2 & -1,7 & -6,5 & -4,5 & -31,9 \\ \text { Kolumbien } & -0,6 & 0,0 & -0,5 & -1,9 & -1,4 & -1,9 & -1,9 & -7,0 \\ \text { Chile } & 0,2 & -0,7 & -1,0 & -0,7 & -0,8 & -0,3 & -1,1 & -6,4 \\ \text { Ecuador } & -0,7 & -0,8 & -0,2 & 0,2 & -0,3 & -0,2 & -0,5 & -3,0 \\ \text { Panama } & 0,1 & -2,1 & -0,0 & -0,2 & -0,6 & -0,3 & 0,1 & -2,8 \\ \text { Uruguay } & -0,3 & -0,2 & -0,1 & -0,1 & -0,3 & -0,4 & -0,4 & -2,5\end{array}$

Lateinamerika u. Karibik

$\begin{array}{lllllllll}\text { insgesamt } & -26,9 & -32,3 & -22,8 & -16,3 & -28,8 & -27,3 & -18,9 & -223,6\end{array}$

Die Angaben für 1990 sind vorläufige Schätzungen.

Quelle: CEPAL, 2.2.O., Santiago 1985-1990

Es ist also nicht mehr die Marktwirtschaft der betroffenen Länder, in der über die Produktion und Verteilung entschieden wird, vielmehr erzwingt die internationale Konkurrenz um Kapital und Kredite auf den internationalen Finanzmärkten, daß Exportüberschüsse erwirtschaftet werden, deren Erlöse für den Schuldendienst verwendet werden können. Jede Bemühung um soziale Gerechtigkeit und ökologische Vernunft stößt sich zuerst einmal daran, daß die Bedienung der Gläubiger absolute Priorität genießt. Und wo deren Marktmacht nicht ausreicht, um ihre Interessen durchzusetzen, müssen die Institutionen helfen, die einst geschaffen wurden, um die Freiheit der Finanzmärkte zu garantieren. So hat beispielsweise der von den Industrielăndem kontrollierte IWF 1982 die chilenische Regierung gezwungen, die privaten chilenischen Auslandsschulden zu ubbernehmen und damit gegen jedes neoliberale Credo die von der internationalen Finanzwelt eingegangenen Risiken zu sozialisieren.

\section{Märkte und Märkte}

Die forcierte Durchsetzung marktwirtschaftlicher Ordnung durch Privatisierung staatlicher Funktionen und Liberalisierung des Außenhandels scheint alle Marktwirtschaften in gleicher Weise zu engyerflochtenen Teilbereichen eines großen allumfassenden Weltmarktes zu machen. Aber auch hier ist das Gegenteil richtig: Der Weltmarkt besteht in Wahrheit aus verschiedenen Märkten, deren Zugang in sehr unterschiedlichem Ausmaß frei ist. Während der Kapitalmarkt immer größere 
Freiheiten der internationalen Konkurrenz erlebt und die Dollar-Milliarden binnen Sekunden um den Erdball kreisen, sind die Markte vieler Länder und Regionen gegen die Einfuhr von bestimmten Gütem und Dienstleistungen sorgfältig abgeschottet. Und es ist nicht zufällig, welche Regionen für welche Güter immer noch dank ihrer Marktmacht auf dem Weltmarkı Einfuhrbeschränkungen durchsetzen können. Wo die Länder Lateinamerikas und überhaupt der Dritten Welt - wie etwa bei Agrarprodukten oder in der Stahlindustrie - eine Chance hätten, sich in der internationalen Konkurrenz gegen die Produzenten aus den kapitalistischen Industrieländern durchzusetzen, da greift trotz jahrelanger zäher GATT-Verhandlungen ein gnadenloser Protektionismus dieser Industrienationen zu ihren eigenen Gunsten ein. Die dadurch erreichte Überproduktion wird dann als »Entwicklungshilfe« oder gar "Katastrophenhilfe « verschenkt oder zu Dumpingpreisen in die Markte der Dritten Welt gepreßt, was die Aufgabe der lateinamerikanischen Länder, die nötigen Exportüberschüsse für die Begleichung ihrer Schuldendienstverpflichtungen zu erreichen, noch einmal erschwert.

Ein strategisch eingesetzter Protektionismus, den Dieter Senghaas einst als »selek. tive Dissoziation vom Weltmarkt« zur Voraussetzung von nachholender Entwicklung für die Länder der Dritten Welt erklărt und legitimiert hatte (vgl. Senghaas 1977), wird jetzt zu dem Mittel, durch das die reichen Länder die Exklusivität ihrer Entwicklung sichern. Die Lokomotive fährt nicht mehr mit den Waggons der Zweiten Klasse auf schnellere Gleise, um den Vorsprung der anderen aufzuholen, sondern sie hat die Waggons der Zweiten und Dritten (und Vierten) Klasse abgekoppelt, um mit den Abteilen Erster Klasse umso besser beschleunigen zu können.

Am wirkungsvollsten erweist sich diese selektive Dissoziation vom Weltmarkt auf dem internationalen Teilmarkt der Ware, die gewöhnlich gar nicht in diese Betrachtungen einbezogen wird, nämlich der Ware Arbeitskraft. Was bei den Märkten für Kapital, Güter und Dienstleistungen als das - zu erstrebende - eigentlich Natürliche gilt, nämlich die freie internationale Konkurrenz, ist auf dem Weltarbeitsmarkt im Verlauf des 20. Jahrhunderts systematisch zur Ausnahme gemacht worden, die allenfalls bei Vorliegen ganz spezifischer Qualifikationen - Wissenschaft, Technik, Sport und Kunst - anerkannt wird. Alle anderen Arbeitsuchenden werden zur Konkurrenz auf dem intemationalen Arbeitsmarkt nur zugelassen, sobald und solange in einem Land ein Engpaß besteht, den sie beheben können. Ist aber der Engpaß beseitigt, werden aus den ehemals umworbenen »Gastarbeitern« jetzt unerwünschte Personen, mögen sie nun »illegale Fremde , »unpolitische Asylbewerber«, »Wirtschaftsflůchtlinge« oder neuerdings »Armutsflüchtlinge« genannt werden (vgl. Müller-Plantenberg 1981)。

Diese Segmentierung des Weltmarktes, einerseits in unterschiedliche Märkte mit einem jeweils anderen Grad an freier internationaler Konkurrenz, andererseits in nationale oder regionale Marktwirtschaften, die deshalb auf den Mårkten für Kapital, Güter, Dienstleistungen oder Arbeitskräfte sehr verschieden stark zur internationalen Konkurrenz zugelassen sind, bewirkt schon allein, daß in den 
einzelnen Ländern die Kontrolle über die Koordinaten der eigenen Marktwirtschaft und damit die Fashigkeit zur Regulierung und die Chance zum demokratischen Eingriff in den Markt sehr verschieden groß sind.

Welche Marktwirtschaften sich aber nun von welchen internationalen Märkten abkoppeln dürfen - wie Westeuropa von den internationalen Märkten für Arbeitskråfte und für Agrarprodukle - und welche Marktwirtschaften von welchen internationalen Mårkten ausgeschlossen werden - wie zeitweise Osteuropa von ganz bestimmten Technologien -, darüber entscheiden allein die Regierungen der reichsten Länder. Als Hauptkonkurrenten sind sie nicht nur gleichzeitig Schiedsrichter, sondern auch noch mitten im Spiel zur Änderung der Regeln befugt. Sie sind deshalb die einzigen, die innerhalb dieses halbfreien Weltmarktes noch über ein Minimum an Kontrolle über die Koordinaten der eigenen Marktwirtschaft verfügen und damit im Prinzip für eine soziale und ökologische Orientierung sorgen könnten.

In den lateinamerikanischen Ländern dagegen erleben wir die Marktwirtschaft, wie sie real existiert. Die auch für ein nur normales Funktionieren der inneren Marktkrăfte erforderliche Kontrolle der äußeren Bedingungen ist den Regierungen unmöglich gemacht.

Die über die Privatisierung staatlicher Funktionen und die Liberalisierung des AuBenhandels bewerkstelligte, möglichst totale Unterwerfung unter das ungleiche Spiel auf dem halbfreien Weltmarkt verlangt gebieterisch, daß im internen Verteilungskampf die Interessen in der Reihenfolge ihrer internationalen Marktmacht bedient werden. Deshalb muß unter allen Umständen zuerst dem Kapital die international übliche Rendite oder eine möglichst höhere zugesichert und garantiert werden. Dem Fluchtkapital können nämlich keine Grenzen gesetzt werden, ihm sind die Tore der internationalen Banken weit geöffnet. Die Einkommensschere offnet sich immer weiter, weil die Besitzer von Kapital oder Qualifikation ihre internationale Mobilităt in Marktmacht umsetzen und höhere Einkommen erzwingen können, wăhrend die Masse der nicht beruflich qualifizierten Arbeitslosen keine Chance hat, im reichen Ausland Aufnahme zu finden. Unter diesen Umständen wird den lateinamerikanischen Ländern die intensive Ausbeutung aller menschlichen und natürlichen Ressourcen, also das Gegenteil von sozialer und ökologischer Orientierung, zur Pflicht gemacht.

Jede auf Wachstum zielende wirtschaftspolitische Strategie hat zur Voraussetzung eine noch tiefere Verbeugung vor der Macht des in- und ausländischen Kapitals und einen Panzerschutz gegen das Aufkommen sozialer Gefühle. Wachstum ist nicht nur gefordert, weil man gern etwas umverteilen würde, sondern schon, weil die Zinsen zu bezahlen sind. Politik beschränkt sich auf die Einsicht in die Notwendigkeit des Sachzwangs. 


\section{Demokratie und Demokratie}

Seir langem ist die Abhängigkeit Lateinamerikas von den Zentren des Weltkapitalismus nicht so eindeutig und so sichtbar gewesen wie heute, aber noch nie wurde so wenig davon gesprochen. Die demokratisch gewählten Präsidenten und Regierungen des Subkontinents erheben den Anspruch und erwecken den Anschein unbezweifelbarer Souverånität - und beugen sich vor dem Sachzwang, freiwillig, aus Einsicht in die Notwendigkeit. Von Abhängigkeit zu sprechen gilt nicht mehr als fein.

Nun hat Demokratie ja eigendlich nicht die Funktion, den Sachzwang zu vollziehen, sondern dem Volkswillen Ausdruck zu verschaffen. Und wo der Sachzwang ganz eindeutig den unmittelbaren Interessen der großen Mehrheit entgegensteht, wäre eigentlich die große Revolte zu erwarten, die sich dann auch gegen eine als ungeniigend oder betrügerisch empfundene Demokratie richten würde. Es fehlt auch nicht an Revolten. Die heftigen Unruhen in Caracas vom Februar 1989, die politischen Proteste in Mexiko nach den letzten Präsidentschaftswahlen, die Guerilla-Bewegungen in Peru oder die Streiks in Managua vom Juli 1990 sind Anzeichen einer großen sozialen und politischen Unzufriedenheit bei breiten Bevölkerungsschichten. Aber sie verdecken nicht den anhaltenden Trend eines breiten Siegeszugs der Demokratisierung in (fast) ganz Lateinamerika. In einem Kontinent, in dem vor zehn Jahren Generäle in den meisten Ländern das unbeschränkte Sagen hatten, vergeht heute kaum ein Monat, in dem nicht irgendwo das Volk zur Wahlume gerufen wird. Wer hat daran ein Interesse?

Die Sozialwissenschaftlerinnen und Sozialwissenschaftler, die sich mit den Demokratisierungsprozessen in Lateinamerika beschaftigt haben, haben sich in der Regel auf die Logik der inneren Entwicklung der Militärdiktaturen konzentriert und aus dem sich kumulierenden Legitimationsdefizit die geradezu zwangsläufig sich ergebenden Demokratisierungstendenzen erklärt. Auf diese Art sind viele kluge und differenzierende Analysen entstanden, über denen aber die historischsoziale Bedeutung der Militärdiktaturen nicht verloren gehen darf: Zwischen den Demokratien vorher und hinterher klafft ein himmelweiter Unterschied.

Die lateinamerikanischen Demokratien, die in den sechziger Jahren mit der Hilfe ausländischen Kapitals die Strategie der importsubstituierenden Industrialisierung verfolgten und dann Anfang der siebziger Jahre zusammen mit anderen Ländern der Dritten Welt für die Schaffung einer Neuen Weltwirtschaftsordnung eintraten, haben sich damals unter dem Druck der Wăhlerinnen und Wăhler bemühen müssen, ihrer Marktwirtschaft eine soziale Orientierung zu geben - von ökologischer Orientierung sprach damals noch niemand. Die sozialisierenden Tendenzen der Demokratien bedrohten die freie Bewegung des Kapitals.

Die historisch-soziale Bedeutung der Militärdiktaturen - mit der Ausnahme der peruanischen von Velasco Alvarado 1968-1975 - bestand unter diesen Umständen in der Herstellung der vollen Bewegungsfreiheit des Kapitals, einer völligen oder doch - im Fall Brasilien - weitgehenden Integration in den Weltmarkt und der Ausrottung aller sozialisierenden Tendenzen. Diese liberale Revolution, die zwei- 
fellos in Chile am grünlichsten betrieben wurde, aber in den anderen Diktaturen kaum weniger effektiv funktioniert hat, hatte zum Ergebnis, daB die neu erstandenen Demokratien auf einer völlig neuen Basis operieren, gewissermaßen auf einer tabula rasa. Die heute real existierende Demokratie basiert auf der nackten Marktwirtschaft in ihrer Abhängigkeit vom halbfreien Weltmarkt. Wo der Sachzwang dieser real existierenden Marktwirtschaft voll durchschlägt, bedarf es der Militärs nicht mehr.

\section{Demokratie und Marktwirtschaft - real existierend}

Das hindert nun nicht, daß der Volkswille etwas anderes fordert: Am Beispiel der Präsidentschaftswahlen, die zwischen 1988 und 1990 in den größeren lateinamerikanischen Låndern stattgefunden haben, läß sich zeigen, mit welchen Mitteln jeweils dem Sachzwang zum Sieg über den Volkswillen erst verholfen werden mußte.

In Chile hat eine große Mehrheit der Bevölkerung dem Kandidaten Hernán Büchi, der offensiv für eine Fortsetzung des ultraliberalen Wirtschaftsprogramms der $\mathrm{Pi}$ nochet-Diktatur eintrat, in den Wahlen vom 14. Dezember 1989 eine klare Absage erteilt und den Christdemokraten Patricio Aylwin gewählt. Aber dessen Minister und Wirtschaftsberater sind, egal ob Sozialisten oder Christdemokraten, inzwischen so sehr von der Notwendigkeit der Fortsetzung der bisherigen Wirtschaftspolitik überzeugt, daB trotz allen Redens von sozialer Gerechtigkeit von einem grundsätzlichen Wandel zugunsten der zwei ärmeren Drittel der sozial extrem gespaltenen Gesellschaft kaum gesprochen werden kann (vgl. Müller-Plantenberg 1990).

Fast zur gleichen Zeit wie die Wahlen in Chile fanden die Stichwahlen für die Präsidentschaft in Brasilien statt, bei denen sich der politische Newcomer Fernando Collor, Überraschungskandidat der Rechten, um gegen den Gewerkschaftsführer Lula von der Arbeiterpartei PT überhaupt bestehen zu können, mit dem populistischen Programm eines Saubermanns vom Thatcheristen zum progresssiven Sozialdemokraten zu mausern versuchte. Das Rezept wirkte und brachte ihm knapp den Wahlerfolg und die Präsidentschaft. Schon einen Tag nach seinem Amtsantritt am 15. März 1990 schockte er dann das ganze Land mit der Sperrung aller größeren Bankguthaben und einem in seiner Radikalität und Strenge einzigartigen antiinflationären Sparprogramm (vgl. Fatheuer/Würtele 1986; Fatheuer 1990). So sehr die Anfangserfolge in Richtung auf eine Stabilisierung der Preise auch von großen Teilen der Bevölkerung begrüßt wurden, die Hauptlast hatten mit steigender Arbeitslosigkeit und sinkenden Reallöhnen auch hier die großen Massen der von Collor umworbenen »descamisados « (Hemdlosen) zu tragen.

Vollends deutlich wird das Auseinanderklaffen von Wahlprogramm und Amtsführung bei den in letzter Zeit gewählten Präsidenten von Argentinien und Peru. Anfang 1989 setzte sich in Argentinien Carlos Saúl Menem als Kandidat der orthodox-autoritären Mehrheit der Gewerkschaften und anderer nationalistischer 
Strömungen innerhalb der peronistischen Partei in den Prăsidentschaftswahlen gegen den liberalen Kandidaten Eduardo Angeloz mit einem Programm durch, das an populistischer Demagogie kaum zu übertreffen war. Aber schon die Regierungsbildung im Juli 1989 zeigte die wahren Intentionen Menems: Fuir die wirtschaftspolitischen Ressorts wurden wichtige Vertreter des transnationalen Konzerns Bunge \& Born gewomen, der stets einer der Hauptadressaten für die sozialen Anklagen der Peronisten gewesen war. Auch hier begann die neue Präsidentschaft entgegen allen programmatischen Außerungen mit einem kurzfristig durchgesetzten antiinflationären Stabilisierungsprogramm mittels umfassender Liberalisierung der Wirtschaftsbeziehungen, Privatisierung von Staatsbetrieben und Sanierung des Staatshaushalts. Auch hier die zu erwartenden Folgen für die Massen der Bevölkerung: starker Rückgang der Produktion, starker Rückgang der Einkommen, starker Rückgang der Beschäftigung (vgl. Ossoni/Dirmoser 1989; Herzog 1989).

Noch größer war nur die Skrupellosigkeit, mit der Alberto Fujimori, der im April 1990 gegen den offensiv ultraliberal auftretenden Romancier Mario Vargas Llosa zum Präsidenten Perus gewählt wurde, gleich nach seinem Amtsantriłt im Juli 1990 alle seine wirtschaftspolitischen Wahlversprechungen dadurch zur Makulatur erklärte, daß er ebenfalls ein Sanierungsprogramm nach Art eines Überfalls durchsetzte. Obwohl ihm erst seine Wahlpropaganda gegen das von Vargas Llosa vorgeschlagene Schockprogramm und sein Eintreten für eine »schrittweise« Sanierung die Unterstützung durch ehemals linke Wählerinnen und Wähler und damit den Wahlsieg gesichert hatten, stellte Fujimori nach Gesprächen in Japan und beim IWF die gesamte Wirtschaft handstreichartig auf das freie Spiel der Marktkräfte um. Auch hier der Widerspruch zwischen der Hoffnung der Massen auf ein Gelingen der Stabilisierung und der brutalen Realität von Rezession und Elend (vgl. Dirmoser 1989; Goedeking 1990; aus anderer Sicht: Rojas 1990).

In Mexiko ist die herrschende politische Klasse, die ihre Heimat in der institutionalisierten Revolutionspartei PRI hat und mit ihrem 1988 an die Regierung beförderten Präsidenten Carlos Salinas de Cortari heute ebenfalls auf die neoliberalen Rezepte des IWF schwơrt, erst gar nicht das Risiko eingegangen, ihr Schicksal einem Kandidaten anzuvertrauen, der wie Cuauhtemoc Cárdenas mit einem teils sozialdemokratischen, teils populistischen Programm zu den Wahlen angetreten war. Nach allen glaubwürdigen Berichten wurden hier direkte Wahlfälschungen in einem Ausmaß betrieben, das den Schluß zuläBt, daß Cårdenas, der Sohn des legendären Präsidenten Lázaro Cárdenas ( 1934-1940), um den möglichen Sieg betrogen wurde (vgl. Reimar 1989).

In Nicaragua schließlich hat der Sandinistischen Befreiungsfront FSLN auch die seit 1985 eingeleitete Wirtschaftspolitik einer Übernahme der IWF-Rezepte nicht mehr genützt. Unter dem Druck von Krieg gegen die Contra und Wirtschaftsblockade wåhlte eine Mehrheit der Bevölkerung am 25. Februar 1990 zur Überraschung aller Beteiligten Violeta Chamorro als Vertreterin der bisherigen Opposition zur Präsidentin des Landes. Wenn das Sanierungsprogramm schon unaus- 
weichlich ist, dann doch bitte ohne Krieg von innen und außen, so lautete die Botschaft (vgl. Rediske 1990).

Die Forderung nach mehr sozialer Gerechtigkeit und nach Schutz gegen wirtschaftspolitische Schockprogramme, die die soziale Ungleichheit sogar noch bewuBt fördern, ist in allen diesen Wahlen nicht nur präsent gewesen, sondern auch in den Wahlkämpfen von den Mehrheiten erhoben worden. Aber wie auch immer, durch äußeren Druck wie im Fall Nicaragua, direkte Wahlfalschung wie in Mexiko, indirekten Wahlbetrug wie in Peru, Argentinien und Brasilien oder allmähliche Anpassung wie im Fall Chile sind diese Mehrheiten um ihren Sieg gebracht worden, damit der Sachzwang der Marktgesetze seine volle Wirkung entfalten kann.

Wo aber die Spannung zwischen Demokratie und Markt, von der oben die Rede war, so aufgelöst wird, daß das Mehrheitsprinzip hinter dem Rücken der Wählenden außerkraftgesetzt wird, weil die so oder so gewählten Repråsentanten von seiner Respektierung das Chaos erwarten, da ist zu fürchten, daB das Vertrauen der Wăhlerinnen und Wähler in die Macht ihrer Wahlstimmen schwindet. Nicht zufällig warnen heute gelegentlich schon manche lateinamerikanischen (linken) Intellektuellen vor demagogischen Forderungen nach sozialer Gerechtigkeit wegen der damit verbundenen Gefahren für die frisch errungene Demokratie.

Dabei wissen die Massen der Bevölkerung durchaus, daß die einzige reale Alternative zur politischen Demokratie nur eine Diktatur ist, von der sie keine Verbesserungen ihrer wirtschaftlichen Situation, wohl aber politische Unterdrückung und Menschenrechtsverletzungen zu erwarten haben. Sie werden deshalb immer noch hoffen und dafür eintreten, daß die innere Logik der Demokratie eines Tages doch noch zu mehr sozialer Gerechtigkeit führt.

Der organisierte Massenbetrug läßt sich nicht beliebig häufig wiederholen. Bleibt die Marktwirtschaft in Lateinamerika so, wie sie heute als System sozialer Apartheid real existiert, dann erhalten die demokratischen Erwartungen der Massen zwangslåufig explosiven Charakter. Die Erste Welt, die den halbfreien Weltmarkt reguliert und organisiert, der diese Marktwirtschaften einschnürt, wird von den Erschuitterungen nicht unberührt bleiben. Durch die Ergebnisse der Massenmigration von »Wirtschaftsflüchllingen « und der Drogenproduktion hat sie bisher nur einen ersten Eindruck davon bekommen, welche Auswege die Mehrheit der Wellbevölkerung ersinnen mag, um an den Früchten der Marktwirtschaft dort, wo sie floriert, teilzuhaben.

\section{Literatur}

Dimoser, D. (1990): Peru: Ein katholischer Japaner und die grotestantische Ethik, in: Dirmoser, D. u.a. (Hrsg.): Von Elend der Metropolen. Lateinamerika. Analysen und Berichte 14, Hamburg

Ehrke, M. (1986): Jenseite der Verschuldungskrise. Uherlegungen zur wirtschaftlichen Zukunf: Lateinamerikas, in: Dirmoser, D. u.a. (Hrsg.): Aussichen auf die Zukunfi. Lateinamerikz. Analysen 
und Berichte 10, Hamburg

Fatheuer, Th.Würtele, W. (1990): Brasilien: Mil Tatkrafi und Demagogie, in: Dimoser, D. u.a. (Hrsg.): Vom Elend der Metropolen. Laleinamerike, Hamburg

Goedeking, U. (1990): Fujimori: Der Mythos zerplatzt, in: Lateinamerika-Nachrichten Heft 197, Berlin Herzog, R. (1989): "Ni Yanquis, ni marxistas" - neoliberalistas!, in: Lateinamerika-Nachrichte" Heft 185, Berlin

Hinkelammen, F. (1989): Der Schuldenautomatismus - Ein Marshallplan für die Erste Welt, in: Dirmoser, D. u.a. (Hrsg.): Verlieyer der Weliwirtschaft. Lateinamarika. Analysen und Berichte 13, Hamburg

Müller-Plantenberg, U. (1981): Einkommensstruktur und Arbeitsmarkt intemational, in: PROKLA Nr. 42. Berlin

Müller-Plantenberg, U. (1990): Chile: Am Ende der liberalen Revolurion, in: Dimoser, D. u.a. (Hrsg.): Vom Elend der Metropolen. Lateinamerika. Analysen und Berichse 14, Hamburg

Ossoni, F./Dimoser, D. (1990): Argentinien: Mir Menem ins Chaos, in: Dimoser, D. u.a. (Hrsg.): Vom Elend der Metropolen. Lateinamerika, Hamburg

Rediske, M. (1990): Nicaragua: Die Niederlage der Avantgarde-Partei, in: Dirmoser, D. u.a. (Hrsg.): Vom Elend der Metropolen. Lateinamerikc. Analysen und Berichte 14, Hamburg

Reimar, P. (1989): Mexiko: Der lange Sommer der demokratischen Opposition, in: Dirmoser, D. u.\&. (Hrsg.): Verlierer der Welkwirtschaft. Lateinamerika. Analysen und Berichte 13, Hamburg Rojas, C. (1990): Fujimori aus anderer Sicht, in: Lateinamerika-Nachrichten Heft 197, Berlin Senghaas, D. (1977): Weltwirtschaftsordnung und Entwicklurgspolitik. Plädoyer für Dissoziation, Frankfurt 